\title{
Open science practices and risks arising from misuse of biological research
}

\author{
James A. Smith ${ }^{1,2}$ and Jonas B. Sandbrink ${ }^{3,4}$
}

1. Botnar Research Centre and Centre for Statistics in Medicine, Nuffield Department of Orthopaedics, Rheumatology and Musculoskeletal Sciences, University of Oxford

2. National Institute for Health Research Oxford Biomedical Research Centre, John Radcliffe Hospital, Oxford

3. Nuffield Department of Medicine, University of Oxford

4. Future of Humanity Institute, University of Oxford

\section{Correspondence: james.smith2@ndorms.ox.ac.uk and jonassandbrink@gtc.ox.ac.uk}

\begin{abstract}
The proliferation of open science may inadvertently increase the chance of deliberate or accidental misuse of research. Here, we examine the interaction between open science practices and biosecurity and biosafety to identify risks and opportunities for risk mitigation. We argue that open data, code, and materials may increase risks from research with misuse potential, despite their general importance. For instance, increased access to protocols, datasets, and computational methods for viral engineering may increase the risk of release of enhanced pathogens. For this dangerous subset of research, both open science and biosecurity goals may be achieved by using access-controlled repositories or application programming interfaces. The increased use of preprints could challenge any strategy for risk mitigation that relies on assessment at the publication stage, emphasising the need for earlier oversight in the research lifecycle. Preregistration of research, a practice promoted by the open science community, provides an opportunity for achieving biosecurity risk assessment at the conception of research. Open science and biosecurity experts have an important role to play in enabling responsible research with maximal societal benefit.
\end{abstract}




\section{Introduction}

Open science aims to increase the reliability and efficiency of scientific research[1,2]. Despite the importance of work to improve scientific practice, increased openness may increase the chance of deliberate or accidental misuse of research. These concerns are particularly salient for biological research on pathogen synthesis and engineering.

Here, we examine how certain open science practices interface with risks arising from the potential misuse of biological research. For the majority of biological research, increased openness is likely to improve our ability to deal with biological threats through improving the efficiency and reliability of science. However, the subset of research in which biological risks may be increased should not be ignored, and tools and systems to encourage and facilitate responsible access to scientific findings must be available. Collaboration between the open science community and the biosecurity and biosafety communities may be mutually beneficial, and will allow more consideration of the potential risks and opportunities associated with open science. Addressing risks and opportunities raised in this paper should help to reduce threats that could undermine the significant progress made in open science to date and have catastrophic consequences for society.

\section{Risks from biological research}

Natural pandemics have posed major threats to human populations throughout history. In recent decades, research in immunology, virology, and other biomedical domains has greatly improved global pandemic preparedness. However, some life science research has the potential to be misused. Misuse risks can be classified into biosafety risks, concerning accidental exposure and release, and biosecurity risks, concerning deliberate misuse[3]. As biotechnology grows more powerful and accessible[4], risks from accidental or deliberate misuse of research may increase.

There are numerous examples of high-profile biosafety incidents[5]. The $1977 \mathrm{H} 1 \mathrm{~N} 1$ influenza epidemic likely resulted from vaccine trials in the Soviet Union, or accidental release from a laboratory, reintroducing a strain that was circulating in the 1950s[6,7]. In 2015, the United States realised that it had distributed live anthrax, as opposed to the intended inactivated anthrax, in 575 shipments to eight countries over a decade[8]. Laboratory accidents involving dangerous pathogens happen frequently and, given the leading cause of such accidents is human error, are difficult to mitigate completely even in high level biocontainment facilities[9]. For instance, in 2003 and 2004, incidents in three different labs researching or containing SARS-CoV-1 lead to a total of 6 lab-acquired infections which could have sparked further epidemics[10].

Deliberate release of pathogens may take place in the context of biological warfare or terrorism. A historic precedent for the intentional misuse of biological agents is set by the Soviet Union's extensive biological weapons program that involved the synthesis and enhancement of plague, smallpox, and other agents[11]. There have also been terrorist incidents such as the 2001 US anthrax attacks likely conducted by a single perpetrator with training and access to biological research materials, showing that greater access to materials and methods can have tragic consequences when abused[12]. 
Certain life sciences research may be misused and increase the risk from deliberate biological events. For example, though advances in viral engineering may be important in areas like vaccine design and cancer therapy, they could be applied to engineer pathogens with increased virulence or transmissibility. Deliberate release of such pathogens could result in a pandemic of unprecedented severity. Research with the greatest misuse potential has been labelled dual-use research of concern (DURC), defined by the National Institutes of Health in the U.S. as "life sciences research that, based on current understanding, can be reasonably anticipated to provide knowledge, information, products or technologies that could be directly misapplied to pose a significant threat with broad potential consequences to public health and safety"[13]. According to the Global Health Security Index, only $1 \%$ of countries have appropriate oversight for potential dual-use life science research with especially dangerous pathogens[14]. The few existing frameworks are limited in scope; for instance, the U.S. DURC policies only apply to research on 15 select agents and toxins at federally-funded institutions[15,16].

Today, information hazards,[17], i.e. knowledge and insights that can cause harm, are frequently associated with greater dual-use potential than the physical products of research[18,19]. Advances in molecular biology, including DNA synthesis and gene editing, continue to lower the barrier to the synthesis and engineering biological agents[20]. Therefore, publicly available information that may inform the engineering of pandemic pathogens and other biological threats are accessible to a growing community. As biotechnology advances, it is increasingly important to effectively mitigate risks from research information with dual-use potential.

\section{Open science and risks from biological research}

'Open science' has been advanced[21-23] in part to address widespread problems identified across the sciences[24]; however, there is little consensus on what the term, or related terms such as 'reproducibility', mean[25-27]. We consider open science to be a set of practices that aim to improve the reliability and efficiency of scientific research[23,28] that are generally characterised by increased transparency. We consider that open science achieves its aims through three instrumental mechanisms: verification, reuse, and accessibility. By verification, we mean the ability to review and critique aspects of the research to establish that what is described corresponds to what was done. An example of this is computational reproducibility, i.e. ensuring the data and code achieve the claimed results. By reuse, we mean the ability to use research outputs for the same or another purpose, such as conducting a replication study, validation study, or secondary analysis. By accessibility we mean making research outputs widely, and usually publicly, available. Accessibility in many cases facilitates efforts to verify and reuse research results.

Open science may contribute to mitigating biosecurity and biosafety risks. Reliable and efficient science is important for effectively preventing and responding to pandemic risks: for example, in developing drugs, vaccines and diagnostics, and implementing effective public health responses. Preprints may have played an important role in scientific and public engagement with COVID-19 research[29]. Research excellence and ethical research conduct, both encouraged by open science, are pillars of responsible life science research for global health security[30]. The move towards open science has involved a cultural shift related to conduct and sharing and research; a similar cultural shift may be required to 
encourage responsible research conduct and sharing to protect the life sciences from misuse. Open science may therefore represent a useful case-study[31].

As we highlight, however, there are instances where open science may exacerbate biosecurity and biosafety risks. Addressing such risks will inevitably reduce the ability to verify, reuse, or access research. However, this need not necessarily reduce reliability and efficiency. When typical open science practices seem inadvisable, we provide tentative suggestions for how reliability and efficiency can still be improved. We focus on three practices that appear relevant to biological risks: open code, data and materials; preprint publication; and preregistration ${ }^{1}$ (Table 1).

\footnotetext{
1 These reflect, for example, the three options provided in the "Conduct your own open science" section on the Centre for Open Science home page: https://www.cos.io/
} 
Table 1: Summary of risks arising from open science practices, strategies to mitigate these risks, and opportunities to improve biosecurity and biosafety.

\begin{tabular}{|c|c|c|c|}
\hline $\begin{array}{l}\text { Open science } \\
\text { practice }\end{array}$ & Possible risks & Mitigation & Opportunity \\
\hline $\begin{array}{l}\text { Open code, data, and } \\
\text { materials }\end{array}$ & $\begin{array}{l}\text { Datasets with dangerous } \\
\text { information more accessible } \\
\text { - Access to computational } \\
\text { methods which may be } \\
\text { misused, including to } \\
\text { interpret datasets } \\
\text { - Increased access to and } \\
\text { misuse of research } \\
\text { methods and tools }\end{array}$ & $\begin{array}{l}\text { - Access control for code, data, or } \\
\text { materials } \\
\text { - } \quad \text { Code-only access to worrying datasets } \\
\text { - Application programming interfaces to } \\
\text { allow widespread and controlled } \\
\text { access to data and computational } \\
\text { models } \\
\text { - Reject posting of material that has } \\
\text { dual-use potential }\end{array}$ & $\begin{array}{l}\text { Differential sharing of material based } \\
\text { on risk } \\
\text { - Create or identify access-controlled } \\
\text { repositories suitable for sharing } \\
\text { protocols } \\
\text { - Improve adherence to FAIR principles } \\
\text { for research outputs through curated } \\
\text { repositories }\end{array}$ \\
\hline Preprint publication & $\begin{array}{l}\text { - } \quad \text { Removes gatekeeper role } \\
\text { that journals may play } \\
\text { - Increases accessibility to } \\
\text { research }\end{array}$ & $\begin{array}{l}\text { Oversight earlier in research process } \\
\text { than publishing stage } \\
\text { - Reject posting of material that has } \\
\text { dual-use potential }\end{array}$ & $\begin{array}{l}\text { - Research on existing policies and } \\
\text { dynamics of preprint vs. journal } \\
\text { publication needed } \\
\text { - Possible coordination among preprint } \\
\text { servers for screening research }\end{array}$ \\
\hline Preregistration & - Minimal risk & $-\quad N / A$ & $\begin{array}{l}\text { - Oversight and risk assessment earlier } \\
\text { in research lifecycle } \\
\text { - Advisory peer review in registered } \\
\text { report-like format } \\
\text { - Pilot new exploratory preregistration } \\
\text { formats in high-risk areas }\end{array}$ \\
\hline
\end{tabular}




\section{Open code, data and materials: a challenge for mitigating misuse}

Sharing code and data allows the research community to reproduce and check analytical findings[32,33] and facilitates reuse. Code is typically shared via repositories such as the Open Science Framework, GitHub, Zenodo, or in supplementary files accompanying a published article. Data may be shared similarly, though discipline-specific repositories[34] are also common. By open materials, we mean detailed, recipe-like explanations (such as written instructions or videos) on how to do certain procedures ${ }^{2}$. Such materials can be shared in supplementary files, dedicated repositories like protocols.io or bio-protocol.org, general purpose repositories like Open Science Framework, or as standalone journal articles (e.g.[36]).

Code could be used directly or adapted to achieve nefarious goals[37]. Machine learning-guided engineering of antibiotic resistance genes exemplifies this: a model for engineering Escherichia coli $\beta$-lactamase has been described and shared openly[38]. TEM-1 $\beta$-lactamase is a well-known antibiotic resistance gene that facilitates resistance of Gram negative bacteria to a range of key antibiotics, including penicillins and cephalosporins[39]. The model allows anyone with access to a computer, in theory, to generate "better-than-natural"[38] variants of TEM-1 $\beta$-lactamase. The authors claim that their work "demonstrates a generalisable and scalable paradigm for low $\mathrm{N}$-protein engineering", where low- $\mathrm{N}$ protein engineering refers to the minimisation of the amount of laboratory work needed to apply the approach to other protein engineering tasks. If these claims are accurate, the machine learning model in question may be applied to the design of antibiotic resistance genes to make bacteria hyper-resistant, including against last-resort antibiotics. Openly shared computational methods may therefore make pathogen engineering more accessible by reducing or even removing the need for laboratory expertise and equipment.

Data may also be associated with misuse risks. The publication of experiments and genetic changes required to make avian influenza transmissible in mammals has previously raised concerns around the security risk of publishing experimental data[40]. Publicly available blueprints for particularly concerning pathogens, such as the genome of the 1918 pandemic influenza virus, feature growing potential for misuse given increasing access to viral synthesis capabilities. More powerful experimental methods mean that increasingly comprehensive datasets are generated with greater potential for misuse. For instance, in high-throughput experiments a virus may be mutated thousands of times and the effect on functions such as immune evasion or binding to human cell surface receptors recorded. Such work has been conducted on pathogens with pandemic potential including SARS-CoV-2 and influenza virus[41,42]. While these experiments are important for vaccine design, the publicly available datasets could be used by malicious actors to inform the enhancement of pandemic pathogens. Beyond the generation of datasets with greater potential for misuse, improved computational methods mean that data can be more effectively used for malicious bioengineering.

\footnotetext{
${ }^{2}$ We limit discussion to digital rather than physical materials because physical materials are already more highly regulated and different considerations apply to them. However, physical materials are included in some definitions of open materials[35].
} 
Publication of detailed methods, for example for the synthesis and engineering of pandemic pathogens, may also increase the risk of accidents and misuse. Detailed protocols may lower the tacit knowledge required to perform certain procedures, making them more accessible to bad actors, inappropriately qualified personnel, or personnel working in inappropriate facilities[43]. A recent protocol detailing how to synthesise recombinant SARS-CoV-2 exemplifies this[44]. The described "reverse genetic system can be used to rapidly engineer viruses with desired mutations to study the virus in vitro and in vivo" and "enable researchers from different research backgrounds to master the use of the reverse genetic system".

Given the misuse potential of research objects like code, datasets, and protocols, approaches for risk mitigation are needed. Across digital research objects, there appears to be a trend towards increased modularisation, i.e. sharing information in dedicated, purpose built repositories, in contrast to supplementary materials. This modularisation may allow differential access to research products according to the risk that they represent. Curated repositories with greater access control could be used that allow reuse and verification when full public disclosure of a research object is inadvisable. Such repositories are already critical for life sciences that deal with personally identifiable information. Peer reviewers could be given access during the article submission process, and subsequent access controls could be defined based on the perceived risk of the information. When information cannot be shared publicly, access controlled repositories would allow researchers to get credit for digital research object curation and creation through publication of persistent and citable meta-data[45]. The Harvard Dataverse (https://dataverse.harvard.edu/) is an example of an existing repository which allows archiving of data and code with customisable restrictions and searchable meta-data to facilitate discoverability. We are not aware of repositories dedicated to protocols that allow access controls despite their potential value. Private workspaces, such as those available at protocols.io, may be suitable with some adaptation.

Non-public clinical datasets may represent a useful model for concerning biological datasets. Participant level data often cannot be shared openly due to concerns with anonymity, so databases that regulate access are required. Clinical Practice Research Datalink (CPRD), for example, collects a range of primary healthcare data from general practices across the UK[46]. Access to data is provided through a research data governance process which includes screening of applicants and review of a protocol. Access is only granted to bona fide researchers with trustworthy funders, and researchers must "have viable plans that maintain public and professional trust, ensure the research is of public benefit, and are methodologically robust" (https://cprd.com/Data-access). There is a thriving open science community with extensive code sharing related to analysis of such datasets, facilitated by use of standardised data formats[47]. Unlike CPRD, one of the leading influenza and coronavirus sequence sharing platforms, GISAID (www.gisaid.org), requires agreement to a Database Access Agreement, but that agreement does not have any statement regarding the purpose of the research. More comprehensive agreements may be warranted when there are concerns over data misuse. 'Code only access', where analytic code is run on datasets but researchers do not see the data directly[48,49], is another possibility.

If widespread access to code or data is desirable, an application programming interface (API; the "mechanism by which users communicate with computers, code and databases in an automated way"[50]) could be used so that certain model functions or data can be freely 
accessed while use for nefarious purposes is prevented. In the context of datasets, APIs have been recommended, for example, for public health bioinformatics[50]. The risk-aware rollout of the OpenAI API platform for the GPT-3 language model provides precedent. Access was initially limited while risks were assessed and, based on the assessed risks, the API continues to limit use and imposes safety standards on third-party applications[51].

The use of APIs and access-controlled repositories may have benefits beyond improved security. The "FAIR Guiding Principles" identify four principles for shared research objects: they should be findable, accessible, interoperable and reusable for machines and people[52]. Though these principles have largely been applied to data, they are intended to apply to other digital research objects including research software[53]. APIs may increase reusability of computational models and aspects of accessibility (i.e. ability to actually use a model[53]). Code is often difficult to run due to dependencies, computing power requirements, or need for specialist skills; APIs can make it easy for anyone to use software. Access-controlled repositories may facilitate interoperability, reuse and findability through enforcing or encouraging standards for meta-data with common vocabularies and appropriate documentation; much data that is available openly is currently challenging to use and find. It is worth noting that the "A" of FAIR, accessibility, is often qualified: "as open as possible and as closed as necessary"[54]. Limiting access on the basis of security or safety concerns would not necessarily contradict this. Developing suitable repositories may therefore provide an opportunity to improve adherence to the FAIR principles, and encourage their adoption across a wider range of research objects.

In the absence of appropriate sharing methods, an immediately implementable recommendation for data, code, and materials repositories is to require a declaration that the submitted information does not have dual-use potential according to a list of criteria, and to reject those that do unless mitigation is in place. When data cannot be shared at all, verification and assessment of reproducibility may still be possible. For example, simulated data, a subset of the data that represents less risk, or data that has been redacted to remove concerning information could be shared. Publication of a checksum with the analysis code is a further option[55].

\section{Preprint publishing: a changing publication landscape offers challenges and opportunities}

The use of preprints - author formatted articles publicly deposited in a repository - in biological and medical sciences has recently increased considerably[56]. Preprints offer a faster route to publishing research than traditional journals and increase the accessibility and ability to rapidly reuse research. There are proposals for funders to mandate preprint posting[57], and several journals now act as 'overlay journals', collating and reviewing articles that have already been published as preprints[58,59]. A key difference compared to journal articles is that some preprint servers do not screen scientific articles before they are made publicly available: in one analysis, $68 \%$ provided some form of screening or moderation before the article was made public[60]. Three popular servers for biological research screen all submissions before posting, though the scope of that screening differs (Box 1). 
Box 1: Preliminary investigation into preprint policies reveals potential gaps

We investigated the policies for screening preprints at three major preprint servers bioRxiv, medRxiv, and arXiv - based on publicly available information. We read submission guidelines, frequently asked questions, and moderation policies, and searched for the server names along with terms like "dual-use" and "dangerous" to identify other relevant information.

- MedRxiv submissions are screened for "material that could potentially endanger the health of individual patients or the public" which "may include, but is not limited to, studies describing dual-use research"[61]. When launched in 2019, a co-founder of medRxiv stated that they would "almost certainly not post" studies of pathogens that could cause harm[62] and medRxiv have historically "declined work involving pathogens of pandemic potential"[63]. During the COVID-19 pandemic, however, medRxiv began accepting work on SARS-CoV-2 because "knowledge about viral variants gained from this work should be disseminated rapidly"[63].

- BioRxiv submissions "undergo a basic screening process for ... material that might pose a health or biosecurity risk"[64] and "dangerous"[65] content. We were unable to find further public information on what research would be considered a biosecurity risk or dangerous. However, we have identified several papers describing viral engineering approaches posted on bioRxiv, suggesting a fairly permissive standard.

- ArXiv submissions are moderated, though the explanation of the moderation process[66] does not mention dual-use, safety, security or similar terms. Since the scope of arXiv includes "quantitative biology"[67], this may be an important shortcoming. Articles presenting data or models that were rejected from bioRxiv or medRxiv due to security concerns might be permitted at arXiv. We are aware of at least one example of a paper that was not accepted at bioRxiv due to concerns about public health later being posted on arXiv[68].

The existence of relevant wording for bioRxiv and medRxiv is promising, though the lack of mention of security or safety in arXiv may be an important gap. A more comprehensive study including more servers and details of policy implementation is needed.

Preprints may therefore remove the 'gatekeeper' role that journals could play in mitigating risks from the publication of research with potential for misuse. Authors may select preprint servers that do not screen research. Unlike publishing in particular journals, there is little incentive to post to a particular preprint server, so little reason not to select one that will immediately post the article. Whether this is important depends on the role that journals play in preventing or altering publication of research with potential for misuse. There are examples where journals and editors have been important in evaluating risks from publication: for example, in 2014, information was redacted from two manuscripts about gene sequences of a novel Clostridium botulinum toxin following consultation between editors, authors, and branches of the US government[69,70]. Some journals also request 
that reviewers help to identify dual-use risks[69]. Preprints may therefore increase the probability that dangerous methods or results are described publicly. Preprints challenge any model relying on review by journals at publication[18], emphasising the need for oversight at other stages in the research life-cycle, such as during design and funding. The need to consider preprints in the context of research with dual-use potential has been expressed previously[71].

As recommended for code, data and materials, preprint servers could require a declaration that the posted research does not present dual-use potential, and reject posting of articles that do not provide this. There are few prominent preprint servers in comparison to the number of journals, which may represent an opportunity for coordination. Relatively few parties would need to agree on a policy for it to be implemented universally, or at least across all of the major servers (such as bioRxiv, medRxiv, arXiv, OSF preprints, and preprints.org). If an article is flagged by at least one server as potentially concerning, other servers could agree not to post that article until it was appropriately peer reviewed. Further research into the role that preprints play in influencing biosafety and biosecurity risks, policies currently in place, and proposals to mitigate those risks, would be valuable.

\section{Preregistration: an opportunity for dual-use oversight}

Preregistration means archiving a time-stamped protocol that can be referred back to once a project is completed. This protocol is generally made public. Study registries, such as clinicaltrials.gov for clinical trials or PROSPERO for systematic reviews, represent a minimal form of preregistration where details of the study design and study outcomes are provided. In a stronger form, preregistration involves providing a detailed plan for the conduct and analysis of a study, including possibly the analytic code. Such plans are often registered on the Open Science Framework or As Predicted. Preregistration aims to limit duplication through disclosure of research plans[72] and reduce the prevalence of questionable research practices by clearly distinguishing planned and exploratory analyses[73].

It seems likely that greater consideration of the research before it is started, as encouraged by preregistration, could help to mitigate misuse risks. Currently, biosecurity risk assessment and management is not consistently conducted at any stage throughout the research lifecycle; preregistration could encourage greater consideration of risks at an early stage. Submission platforms could ask researchers to reflect on the dual-use potential of their work. In certain high-risk fields, platforms could request that details of hazard assessment be provided, which could be incentivised by journals requesting evidence of such assessments on publication. A safety and security form is required as part of the International Genetically Engineered Machine (iGEM) competition[74], which may be a useful model. In cases where researchers are unsure or do have concerns, they could be directed to an expert or relevant resources.

Registered reports are a type of preregistration and journal article that may present an opportunity for oversight[75]. In registered reports, the introduction and methods (the stage one submission) are peer-reviewed before the work begins. If the stage one submission is accepted, the authors are guaranteed publication of the complete article (the stage two submission) regardless of the results, provided that they follow the proposed methods and the interpretation of the results is reasonable. Peer review at an earlier stage in the research 
life-cycle represents a clear opportunity. When stage one submissions are received that could present biosecurity or biosafety risks, editors could assign biosecurity experts to risk-assess the article, in parallel with the traditional scientific peer review. This is similar to how registered reports currently operate, with specialists in different areas relevant to the manuscript (e.g. statistics, particular methods) reviewing submissions. A biosecurity report could detail recommendations on how the research could be conducted and reported to minimise risk, or if necessary advise against conduct altogether. In cases where review identifies risks, it remains an open question whether review reports should be made openly available. Sharing may encourage responsible discourse, but some arguments against conducting research may themselves present risk by highlighting how research could be misused.

The format of registered reports and preregistrations is currently focussed on hypothesis-testing, confirmatory research. In general, concerning biological research is more likely to be exploratory, involving discovery or development of tools, and without methods and aims that can be defined adequately in advance. However, there are proposals in the open science community to adapt preregistrations to be suitable for exploratory work[76]. If implemented, early collaboration with biosecurity experts would be advantageous in ensuring that risk of misuse is one of the criteria considered. Proactively encouraging early trials of any new format of advisory peer review in areas of perceived high risk, such as synthetic mammalian virology, as suggested elsewhere[20], could be beneficial. Addressing dual-use risks at the early stages of the research lifecycle may be more effective than suppressing the dissemination of dangerous insights after work is completed. Therefore, interventions aimed at encouraging review at the conception of research seem particularly promising.

\section{Discussion}

We highlight several opportunities for reducing risks from research with dual-use potential. First, increased modularisation of research may facilitate differential sharing of research outputs depending on the risk they represent. There is a need to evaluate the suitability of existing tools that are used for sharing in terms of usability and security. To encourage the maximal adoption of tools that facilitate restricted access when needed, they must be as simple as possible to use from the perspective of both the researchers depositing materials and later users of those materials. They must also be secure. If existing tools are not suitable, new platforms may need to be developed. In either case, encouraging and monitoring the adoption of security-friendly sharing practices will be essential. Second, preprints may remove any gatekeeper role that journals play, as evidenced by the lack of screening by some preprint servers, emphasising the need for oversight throughout the research lifecycle rather than solely at the publication stage. Finally, preregistration and registered reports may encourage greater consideration of dual-use potential early in the research process. Existing preregistration formats will require adaptation to be suitable for this purpose. There is a need for guidance and input from individuals or organisations with experience in assessing research with dual-use potential to guide and pilot those adaptations in relevant communities.

The concerns and proposals discussed here may be relevant to a range of research areas. Aspects of vaccine research, gene therapy research, and cancer therapeutics development 
may be associated with risks[77]. Considerable discussion has taken place in the artificial intelligence community about the potential for misuse of published code, such as in the context of deepfake videos for blackmail[78]. Studies that model terrorist scenarios are available, which might assist bad actors in predicting the impact of attacks. It is difficult to identify and anticipate all concerning research areas and formal regulation cannot keep pace[74]. It is therefore important to consider the possibility of research misuse when developing and implementing new open science tools and initiatives for general use.

Incentives for open science require careful consideration. Many have been proposed, including changing hiring practices to support open data, open materials and preregistration[79-81], open science leaderboards[82], journal scores based on transparency[83], badges acknowledging open science on published papers[84], and assessment of open science practices by funders[85]. These incentives must allow limited disclosure when it is justified on the basis of safety or security concerns. Open data badges, for example, are available "if sensitive, personal, data are available only from an approved third party"[86] but not explicitly when the data exhibits safety or security risk. Researchers must not be penalized for responsible disclosure or incentivised to disclose irresponsibly.

Any proposal to allow researchers to reduce public sharing could be exploited by those unwilling to invest the effort that open science requires. For example, researchers who have inadequately documented or fabricated data might invent security concerns. Alternatives to public sharing must therefore include appropriate verification, perhaps through peer review, to ensure that they are available for reuse when appropriate. Since this may increase the burden on reviewers and editors, there may be a need for individuals or organisations with appropriate expertise who are willing to and capable of providing this service. Funders interested in biosecurity and biosafety could support this.

Common to much discussion in this paper is the need for input from experts in risks of biological research. As these risks appear to be relatively neglected, this may be a key bottleneck in developing and implementing changes. Greater investment in expertise related to biosecurity and biosafety will likely be important for realisation of any proposals involving peer review for risk assessment or mitigation purposes. More generally, consideration of downside risk of both open science and biological science appears to be neglected in comparison to its plausible magnitude. Education and outreach may help to increase awareness among relevant stakeholders.

\section{Conclusion}

Open science practices may in many cases contribute positively to our ability to deal with biological threats. However, given the many concerning examples of biosafety and biosecurity incidents, the potential threat posed by the increasing accessibility and usability of scientific research to all actors cannot be ignored. Increased sharing of code, data and materials in particular are concerning in some cases. There is an urgent need to address the inadvertent risks associated with certain open science practices and encourage responsible sharing and access. For preprints, the lack of screening in some cases challenges strategies relying on assessment of dual-use potential at the publication stage, but interventions may be possible and should be explored. Preregistration is a useful model that could encourage risk assessment and advisory peer review of research with dual-use potential earlier in the 
research lifecycle. In general, there is a need for ongoing, critical evaluation of incumbent and changing scientific practices, and consideration of the risks that such practices represent.

\section{Acknowledgements}

We are grateful to the following people for review of earlier drafts of this manuscript: Nick Bostrom, Verena Heise, Malika Ihle, David Manheim, Joshua Monrad, Cecilia Tilli, and James Wagstaff. This project received support from the Effective Altruism Funds programme via the Long Term Future Fund. James Smith's postdoctoral position is funded by the Oxford National Institute for Health Research Biomedical Research Centre. The views expressed are those of the author(s) and not necessarily those of the NHS, the NIHR, or the Department of Health. Jonas Sandbrink's doctoral research is funded by Open Philanthropy.

\section{Conflict of interest}

None to declare.

\section{References}

1. Wallach JD, Boyack KW, loannidis JPA. Reproducible research practices, transparency, and open access data in the biomedical literature, 2015-2017. PLOS Biology. 2018;16: e2006930. doi:10.1371/journal.pbio.2006930

2. Serghiou S, Contopoulos-loannidis DG, Boyack KW, Riedel N, Wallach JD, loannidis JPA. Assessment of transparency indicators across the biomedical literature: How open is open? PLOS Biology. 2021;19: e3001107. doi:10.1371/journal.pbio.3001107

3. Beeckman DSA, Rüdelsheim P. Biosafety and Biosecurity in Containment: A Regulatory Overview. Frontiers in Bioengineering and Biotechnology. 2020;8: 650. doi:10.3389/fbioe.2020.00650

4. Jackson SS, Sumner LE, Garnier CH, Basham C, Sun LT, Simone PL, et al. The accelerating pace of biotech democratization. Nat Biotechnol. 2019;37: 1403-1408. doi:10.1038/s41587-019-0339-0

5. Manheim D, Lewis G. High-risk human-caused pathogen exposure events from 1975-2016. F1000Research; 2021. doi:10.12688/f1000research.55114.1

6. $\quad$ Palese P. Influenza: old and new threats. Nat Med. 2004;10: S82-S87. doi:10.1038/nm1141

7. Enemark C. Biosecurity Dilemmas: Dreaded Diseases, Ethical Responses, and the Health of Nations. Georgetown University Press; 2017. Available:

https://www.jstor.org/stable/j.ctt1kk672v

8. Sosin DM. Review of Department of Defense Anthrax Shipments. 2015; 13.

9. Klytz L. Human error in high-biocontainment labs: a likely pandemic threat. In: Bulletin of the Atomic Scientists [Internet]. 25 Feb 2019 [cited 15 Aug 2021]. Available:

https://thebulletin.org/2019/02/human-error-in-high-biocontainment-labs-a-likely-pande mic-threat/

10. Demaneuf G. The Good, the Bad and the Ugly: a review of SARS Lab Escapes. Zenodo; 2020 Nov 27. doi:10.5281/zenodo.5091301

11. Gilsdorf JR, Zilinskas RA. New Considerations in Infectious Disease Outbreaks: The Threat of Genetically Modified Microbes. Clinical Infectious Diseases. 2005;40: 1160-1165. doi:10.1086/428843

12. Bush LM, Perez MT. The Anthrax Attacks 10 Years Later. Ann Intern Med. 2012;156: 
41-44. doi:10.7326/0003-4819-155-12-201112200-00373

13. Dual-Use Research | NIH Office of Intramural Research. [cited 28 Jun 2021]. Available: https://oir.nih.gov/sourcebook/ethical-conduct/special-research-considerations/dual-use -research

14. Agenda 2024 GHS. APP3 Statement on Biosecurity and Biosafety During the COVID-19 Pandemic. In: Global Health Security Agenda [Internet]. 30 Jul 2020 [cited 16 Aug 2021]. Available:

https://ghsagenda.org/2020/07/30/app3-statement-on-biosecurity-and-biosafety-duringthe-covid-19-pandemic/

15. United States Government Policy for Oversight of Life Sciences Dual Use Research of Concern. 2012.

16. United States Government Policy for Institutional Oversight of Life Sciences Dual Use Research of Concern. 2014. Available:

https://www.phe.gov/s3/dualuse/documents/oversight-durc.pdf

17. Bostrom N. Information hazards: A typology of potential harms from knowledge.

Review of Contemporary Philosophy. 2011; 44-79.

18. Musunuri S, Sandbrink J, Monrad J, Palmer M, Koblentz G. Rapid proliferation of pandemic research: implications for dual-use risks. mBio. 2021;12. doi:https://doi.org/10.1128/mBio.01864-21

19. Lewis G, Millett P, Sandberg A, Snyder-Beattie A, Gronvall G. Information Hazards in Biotechnology. Risk Analysis. 2019;39: 975-981. doi:10.1111/risa.13235

20. Esvelt KM. Inoculating science against potential pandemics and information hazards. PLOS Pathogens. 2018;14: e1007286. doi:10.1371/journal.ppat.1007286

21. Nosek BA, Alter G, Banks GC, Borsboom D, Bowman SD, Breckler SJ, et al. Promoting an open research culture. Science. 2015;348: 1422-1425.

doi:10.1126/science.aab2374

22. Miguel E, Camerer C, Casey K, Cohen J, Esterling KM, Gerber A, et al. Promoting Transparency in Social Science Research. Science. 2014;343: 30-31. doi:10.1126/science. 1245317

23. Munafò MR, Nosek BA, Bishop DVM, Button KS, Chambers CD, Percie du Sert N, et al. A manifesto for reproducible science. Nature Human Behaviour. 2017;1: 0021. doi:10.1038/s41562-016-0021

24. Baker M. 1,500 scientists lift the lid on reproducibility. Nature. 2016;533: 452-454. doi:10.1038/533452a

25. Goodman SN, Fanelli D, loannidis JPA. What does research reproducibility mean? Science Translational Medicine. 2016;8: 341ps12-341ps12. doi:10.1126/scitranslmed.aaf5027

26. Levin N, Leonelli S, Weckowska D, Castle D, Dupré J. How Do Scientists Define Openness? Exploring the Relationship Between Open Science Policies and Research Practice. Bulletin of Science, Technology \& Society. 2016;36: 128-141. doi:10.1177/0270467616668760

27. Vicente-Saez R, Martinez-Fuentes C. Open Science now: A systematic literature review for an integrated definition. Journal of Business Research. 2018;88: 428-436. doi:10.1016/j.jbusres.2017.12.043

28. Allen C, Mehler DMA. Open science challenges, benefits and tips in early career and beyond. PLOS Biology. 2019;17: e3000246. doi:10.1371/journal.pbio.3000246

29. Fraser N, Brierley L, Dey G, Polka JK, Pálfy M, Nanni F, et al. The evolving role of preprints in the dissemination of COVID-19 research and their impact on the science communication landscape. PLOS Biology. 2021;19: e3000959. doi:10.1371/journal.pbio.3000959

30. Information NC for B, Pike USNL of M 8600 R, MD B, Usa 20894. Responsible Life Sciences Research for Global Health Security. World Health Organization; 2010. Available: https://www.ncbi.nlm.nih.gov/books/NBK305040/

31. Atlas RM, Dando M. The dual-use dilemma for the life sciences: perspectives, conundrums, and global solutions. Biosecur Bioterror. 2006;4: 276-286. 
doi:10.1089/bsp.2006.4.276

32. Baker M. Why scientists must share their research code. Nature. 2016 [cited 17 Aug 2021]. doi:10.1038/nature.2016.20504

33. Goldacre B, Morton CE, DeVito NJ. Why researchers should share their analytic code. BMJ. 2019;367: I6365. doi:10.1136/bmj.16365

34. Scientific Data recommended repositories. figshare; 2019. doi:10.6084/m9.figshare.1434640.v16

35. Ihle M, Bishop D, Fortunato L. Open research at Oxford survey. 2021 [cited 1 Nov 2021]. doi:10.5281/zenodo.4437067

36. Bewley KR, Coombes NS, Gagnon L, Mclnroy L, Baker N, Shaik I, et al. Quantification of SARS-CoV-2 neutralizing antibody by wild-type plaque reduction neutralization, microneutralization and pseudotyped virus neutralization assays. Nat Protoc. 2021;16: 3114-3140. doi:10.1038/s41596-021-00536-y

37. Carlson CJ, Farrell MJ, Grange Z, Han BA, Mollentze N, Phelan AL, et al. The future of zoonotic risk prediction. Philosophical Transactions of the Royal Society B: Biological Sciences. 2021;376. doi:10.1098/rstb.2020.0358

38. Biswas S, Khimulya G, Alley EC, Esvelt KM, Church GM. Low-N protein engineering with data-efficient deep learning. Nat Methods. 2021;18: 389-396. doi:10.1038/s41592-021-01100-y

39. Salverda MLM, De Visser JAGM, Barlow M. Natural evolution of TEM-1 $\beta$-lactamase: experimental reconstruction and clinical relevance. FEMS Microbiol Rev. 2010;34: 1015-1036. doi:10.1111/j.1574-6976.2010.00222.x

40. Berns KI, Casadevall A, Cohen ML, Ehrlich SA, Enquist LW, Fitch JP, et al. Adaptations of Avian Flu Virus Are a Cause for Concern. Science. 2012;335: 660-661. doi:10.1126/science.1217994

41. Lee JM, Eguia R, Zost SJ, Choudhary S, Wilson PC, Bedford T, et al. Mapping person-to-person variation in viral mutations that escape polyclonal serum targeting influenza hemagglutinin. Lipsitch M, Kirkegaard K, Lipsitch M, editors. eLife. 2019;8: e49324. doi:10.7554/eLife.49324

42. Starr TN, Greaney AJ, Hilton SK, Crawford KHD, Navarro MJ, Bowen JE, et al. Deep mutational scanning of SARS-CoV-2 receptor binding domain reveals constraints on folding and ACE2 binding. 2020 Jun p. 2020.06.17.157982. doi:10.1101/2020.06.17.157982

43. Pannu J, Sandbrink JB, Watson M, Palmer MJ, Relman DA. Protocols and risks: when less is more. Nat Protoc. 2021; 1-2. doi:10.1038/s41596-021-00655-6

44. Xie X, Lokugamage KG, Zhang X, Vu MN, Muruato AE, Menachery VD, et al. Engineering SARS-CoV-2 using a reverse genetic system. Nat Protoc. 2021;16: 1761-1784. doi:10.1038/s41596-021-00491-8

45. Hrynaszkiewicz I, Khodiyar V, Hufton AL, Sansone S-A. Publishing descriptions of non-public clinical datasets: proposed guidance for researchers, repositories, editors and funding organisations. Research Integrity and Peer Review. 2016;1: 6. doi:10.1186/s41073-016-0015-6

46. Herrett E, Gallagher AM, Bhaskaran K, Forbes H, Mathur R, van Staa T, et al. Data Resource Profile: Clinical Practice Research Datalink (CPRD). International Journal of Epidemiology. 2015;44: 827-836. doi:10.1093/ije/dyv098

47. van Bochove K. Chapter 3 Open Science | The Book of OHDSI. Available: https://ohdsi.github.io/TheBookOfOhdsi/

48. Dayan I, Roth HR, Zhong A, Harouni A, Gentili A, Abidin AZ, et al. Federated learning for predicting clinical outcomes in patients with COVID-19. Nat Med. 2021; 1-9. doi:10.1038/s41591-021-01506-3

49. Williamson EJ, Walker AJ, Bhaskaran K, Bacon S, Bates C, Morton CE, et al. Factors associated with COVID-19-related death using OpenSAFELY. Nature. 2020;584: 430-436. doi:10.1038/s41586-020-2521-4

50. Black A, MacCannell DR, Sibley TR, Bedford T. Ten recommendations for supporting open pathogen genomic analysis in public health. Nat Med. 2020;26: 832-841. 
doi:10.1038/s41591-020-0935-z

51. OpenAI API. In: OpenAI [Internet]. 11 Jun 2020 [cited 30 Aug 2021]. Available:

https://openai.com/blog/openai-api/

52. Wilkinson MD, Dumontier M, Aalbersberg ljJ, Appleton G, Axton M, Baak A, et al. The FAIR Guiding Principles for scientific data management and stewardship. Sci Data. 2016;3: 160018. doi:10.1038/sdata.2016.18

53. Lamprecht A-L, Garcia L, Kuzak M, Martinez C, Arcila R, Martin Del Pico E, et al. Towards FAIR principles for research software. Data Science. 2020;3: 37-59. doi:10.3233/DS-190026

54. Landi A, Thompson M, Giannuzzi V, Bonifazi F, Labastida I, da Silva Santos LOB, et al. The "A" of FAIR - As Open as Possible, as Closed as Necessary. Data Intelligence. 2020;2: 47-55. doi:10.1162/dint_a_00027

55. Van Lissa CJ, Brandmaier AM, Brinkman L, Lamprecht A-L, Peikert A, Struiksma ME, et al. WORCS: A workflow for open reproducible code in science. Data Science. 2021;4: 29-49. doi:10.3233/DS-210031

56. Preprint summary metrics. [cited 16 Aug 2021]. Available: https://rxivist.org/stats

57. Sever R, Eisen M, Inglis J. Plan U: Universal access to scientific and medical research via funder preprint mandates. PLOS Biology. 2019;17: e3000273. doi:10.1371/journal.pbio.3000273

58. Eisen MB, Akhmanova A, Behrens TE, Harper DM, Weigel D, Zaidi M. Implementing a "publish, then review" model of publishing. eLife. 2020;9: e64910. doi:10.7554/eLife.64910

59. Eysenbach G. Celebrating 20 Years of Open Access and Innovation at JMIR Publications. Journal of Medical Internet Research. 2019;21: e17578. doi:10.2196/17578

60. Malicki M, Jeroncic A, ter Riet G, Bouter LM, loannidis JPA, Goodman SN, et al. Preprint Servers' Policies, Submission Requirements, and Transparency in Reporting and Research Integrity Recommendations. JAMA. 2020;324: 1901. doi:10.1001/jama.2020.17195

61. medRxiv Freqeuently Asked Questions. [cited 17 Nov 2021]. Available: https://www.medrxiv.org/about/FAQ

62. Kaiser J. Medical preprint server debuts. Science. 2019; 5.

63. Sever R, Inglis J, Bloom T, Rawlinson C, Krumholz H, Ross J. Pandemic preprints-a duty of responsible stewardship. In: The BMJ [Internet]. 27 Apr 2021 [cited 17 Nov 2021]. Available:

https://blogs.bmj.com/bmj/2021/04/27/pandemic-preprints-a-duty-of-responsible-stewar dship/

64. bioRxiv Submission Guide. [cited 17 Nov 2021]. Available: https://www.biorxiv.org/submit-a-manuscript

65. bioRxiv Frequently Asked Questions. [cited 17 Nov 2021]. Available: https://www.biorxiv.org/about/FAQ

66. arXiv moderation | arXiv e-print repository. [cited 17 Nov 2021]. Available: https://arxiv.org/help/moderation\#what-policies

67. arXiv.org e-Print archive. [cited 17 Nov 2021]. Available: https://arxiv.org/

68. Kwon D. How swamped preprint servers are blocking bad coronavirus research. Nature. 2020;581: 130-131. doi:10.1038/d41586-020-01394-6

69. Hooper DC, Hirsch MS. Novel Clostridium botulinum Toxin and Dual Use Research of Concern Issues. The Journal of Infectious Diseases. 2014;209: 167-167. doi:10.1093/infdis/jit528

70. Relman DA. "Inconvenient Truths" in the Pursuit of Scientific Knowledge and Public Health. The Journal of Infectious Diseases. 2014;209: 170-172. doi:10.1093/infdis/jit529

71. Schloss PD. Preprinting Microbiology. mBio. 2017;8: e00438-17. doi:10.1128/mBio.00438-17

72. ICMJE | Recommendations | Clinical Trials. [cited 17 Jun 2020]. Available: 
http://www.icmje.org/recommendations/browse/publishing-and-editorial-issues/clinical-tr ial-registration.html

73. Nosek BA, Ebersole CR, DeHaven AC, Mellor DT. The preregistration revolution. Proc Natl Acad Sci USA. 2018;115: 2600-2606. doi:10.1073/pnas.1708274114

74. Millett $P$, Alexanian $T$. Implementing adaptive risk management for synthetic biology: Lessons from iGEM's safety and security programme. Engineering Biology. n/a. doi:10.1049/enb2.12012

75. Warmbrod KL, Montague MG, Gronvall GK. COVID-19 and the gain of function debates. EMBO reports. 2021;22: e53739. doi:10.15252/embr.202153739

76. Dirnagl U. Preregistration of exploratory research: Learning from the golden age of discovery. PLOS Biology. 2020;18: e3000690. doi:10.1371/journal.pbio.3000690

77. Sandbrink JB, Koblentz GD. Biosecurity risks associated with vaccine platform technologies. Vaccine. 2021; S0264-410X(21)00171-7. doi:10.1016/j.vaccine.2021.02.023

78. Shevlane T, Dafoe A. The Offense-Defense Balance of Scientific Knowledge: Does Publishing AI Research Reduce Misuse? Proceedings of the AAAl/ACM Conference on Al, Ethics, and Society. New York, NY, USA: Association for Computing Machinery; 2020. pp. 173-179. doi:10.1145/3375627.3375815

79. Schönbrodt F. Changing hiring practices towards research transparency: The first open science statement in a professorship advertisement. In: Nicebread [Internet]. 6 Jan 2016 [cited 2 Jul 2021]. Available: https://www.nicebread.de/open-science-hiring-practices/

80. Ulrich Dirnagl. If you are applying for a professorship at the Charite you now need to tell us about your contributions to your scientific field, open science, team science, interactions with stakeholders. Past and future plans. As a structured narrative. https://t.co/Im3aXBGSE0. In: @dirnagl [Internet]. 4 Mar 2018 [cited 2 Jul 2021]. Available: https://twitter.com/dirnagl/status/970227847943114752

81. Buck S. Beware performative reproducibility. Nature. 2021;595: 151-151. doi:10.1038/d41586-021-01824-z

82. Transparency Audits for Science - Curate Science. [cited 2 Jul 2021]. Available: https://curatescience.org

83. Woolston C. TOP Factor rates journals on transparency, openness. 2020 [cited 2 Jul 2021]. Available:

https://www.natureindex.com/news-blog/top-factor-rates-journals-on-transparency-open ness

84. Kidwell MC, Lazarević LB, Baranski E, Hardwicke TE, Piechowski S, Falkenberg L-S, et al. Badges to Acknowledge Open Practices: A Simple, Low-Cost, Effective Method for Increasing Transparency. PLOS Biology. 2016;14: e1002456. doi:10.1371/journal.pbio.1002456

85. de Jonge H, Cruz M, Holst S. Funders need to credit open science. Nature. 2021;599: 372-372. doi:10.1038/d41586-021-03418-1

86. Approved Protected Access Repositories. In Badges to Acknowledge Open Practices. 2020 [cited 2 Jul 2021]. Available: https://osf.io/tvyxz/wiki/8.\%20Approved\%20Protected\%20Access\%20Repositories/ 